\title{
Silicon nanophotonics using deep-UV lithography
}

\author{
P. Bienstman, F. Van Laere, D. Taillaert, P. Dumon, \\ W. Bogaerts, K. De Vos, D. Van Thourhout, R. Baets \\ Ghent University, Department of Information Technology \\ Sint-Pietersnieuwstraat 41, 9000 Gent, Belgium \\ Peter.Bienstman@UGent.be
}

\begin{abstract}
We will present recent progress in several devices based on silicon-on-insulator nanophotonics using deep-UV lithography. We will report on high efficiency grating couplers, ultra-compact arrayed waveguide gratings and ringresonator based biosensors.
\end{abstract}

Keywords: nanophotonics, arrayed waveguide gratings, ring resonators, biosensors.

\section{INTRODUCTION}

Silicon-on-insulator offers a very interesting platform for nanophotonics because of the high-index contrast and the possibility of integration with CMOS. We report here on several devices fabricated with deep-UV lithography, which opens the way for mass fabrication of these components. We will discuss three types of devices: high efficiency grating couplers using a metal mirror, ultra-compact arrayed waveguide gratings and ring-resonator based biosensors.

\section{HIGH EFFICIENCY GRATING COUPLER WITH METAL MIRROR}

\subsection{Introduction}

Coupling to fiber remains a severe issue in optical communication networks. The ongoing trend to make components smaller in order to integrate them on one chip makes the problem even more difficult. The large difference in dimensions between fiber and waveguides on chip causes high insertion losses and high packaging cost. Key features of effective solutions are compactness, broadband operation, and low insertion loss. Using an inverse taper approach can meet these requirements $[1,2]$. Another attractive solution is the use of grating couplers, since they open the prospect of wafer-scale testing. Light is coupled out-of-plane from fiber to waveguide, and in- and outcoupling can occur anywhere on the chip. Traditional grating couplers use weak gratings and have a narrow bandwidth. We use strong and compact gratings in SOI for butt-coupling with the fiber. The principle is shown in Fig. 1. 


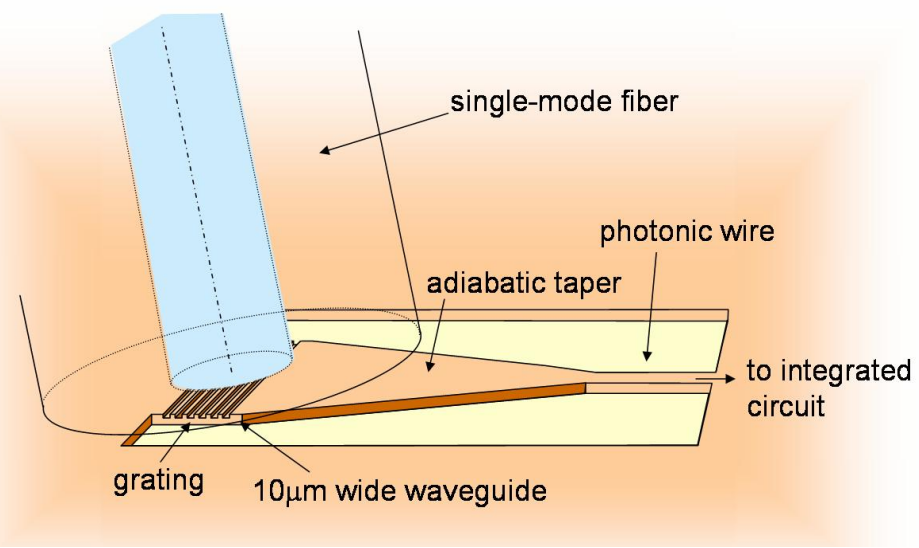

Figure 1 Grating coupler for coupling between single-mode fiber and photonic wires

A coupling efficiency of $33 \%$ for a $10 \times 10 \mu \mathrm{m}^{2}$ grating coupler has been demonstrated in [3]. The coupling efficiency is limited by radiation towards the substrate. This can be avoided by adding a bottom mirror, which reflects the downwards radiated wave. We have added a gold bottom mirror to an existing SOI-grating coupler using BCB wafer bonding [4]. The measured coupling efficiency is $69 \%$.

\subsection{Design}

The couplers are designed with CAMFR [5], an eigenmode expansion tool, for TE-polarization. The fiber is tilted 10 degrees from vertical position in order to avoid $2^{\text {nd }}$ order reflection. We start from an existing SOI grating coupler (Sicore layer $=220 \mathrm{~nm}, \mathrm{SiO}_{2}$ - buried oxide layer $=1 \mu \mathrm{m}$ ) without bottom mirror and with following grating parameters: period $=610 \mathrm{~nm}$, filling factor $=0.5$ and etch depth $=50 \mathrm{~nm}$. The simulated coupling efficiency is $30 \%$. A field plot of the structure is shown in Fig. 2.

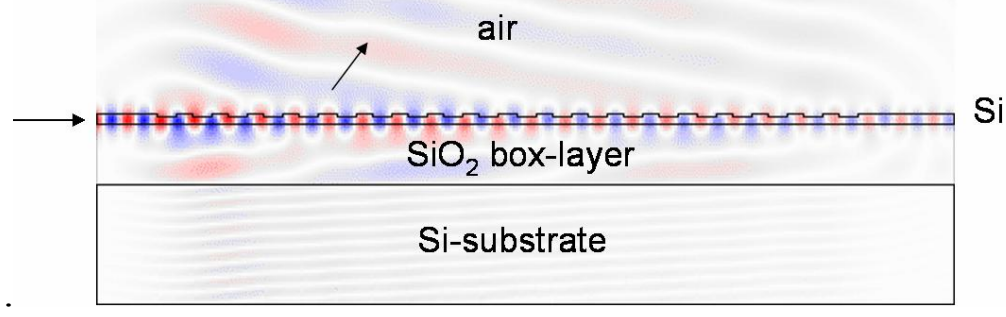

Figure 2 Field plot of an SOI-grating coupler without bottom mirror. An important amount of the power is radiated towards the substrate.

This structure is extended with a gold bottom mirror. A low index layer - in our case BenzoCycloButene (BCB) - has to cover the grating. The optimal thickness of this layer is $840 \mathrm{~nm}$. Then a $50 \mathrm{~nm}$ gold mirror is evaporated, and the structure is bonded with another BCB-layer onto a host-substrate. Finally the Silicon-substrate is removed. The theoretical coupling efficiency to fiber is $72 \%$. A field plot of the structure with bottom mirror is shown in Fig. 3 . 


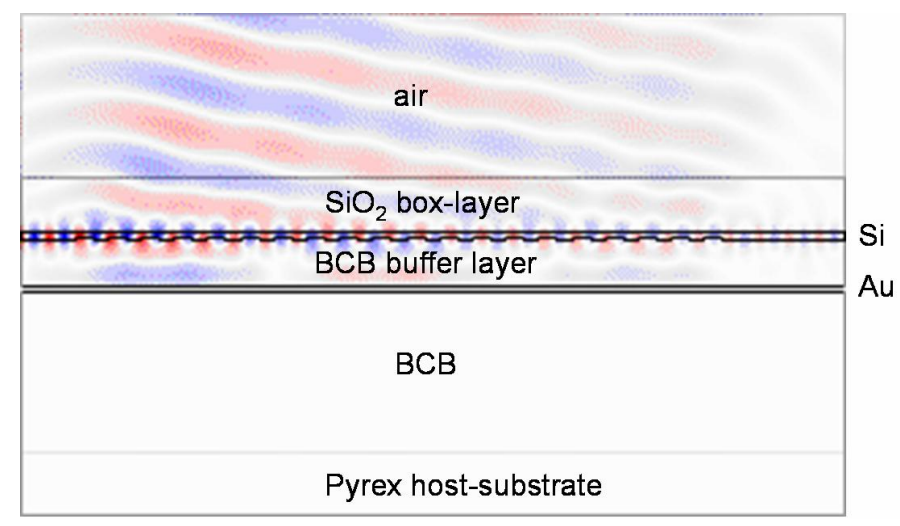

Figure 3 Field plot of an SOI-grating coupler with bottom mirror. No power is radiated towards the substrate.

\subsection{Fabrication}

The SOI-structures are defined with $248 \mathrm{~nm}$ DUV lithography [6]. The waveguides are $10 \mu \mathrm{m}$ wide. We use BenzoCycloButene (BCB), a spin-on polymer from Dow Chemical, both as a low index dielectric and as a bonding agent. First, a BCB-layer with target thickness of $840 \mathrm{~nm}$ is spincoated onto the SOI-die and cured for 40 minutes at 210 degrees. Then, only the grating area is covered with a $50 \mathrm{~nm}$ evaporated gold layer using lift-off. The die is then bonded under vacuum onto a pyrex-substrate using an approximately $3 \mu \mathrm{m}$ thick BCB-layer. The BCB is cured for 1 hour at 250 degrees and the silicon substrate is removed using lapping, $\mathrm{SF}_{6}$ plasma etching and wet etching in $\mathrm{KOH}$. A picture of the fabricated structure and an SEM-picture of a FIB cross-section is shown in Fig. 4.
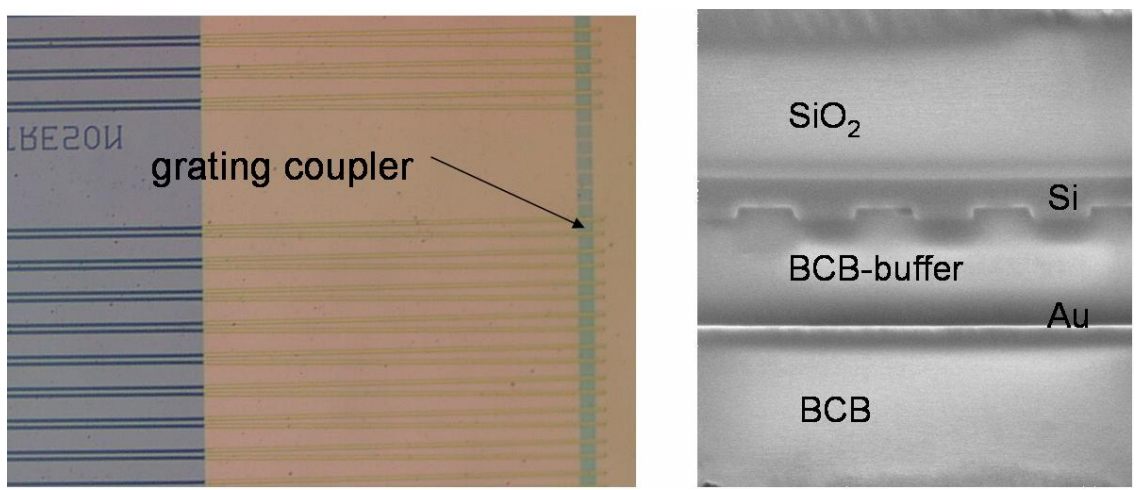

Figure.4 (Left) top view of the fabricated SOI grating coupler with bottom mirror. (Right) SEM-picture of a FIB cross-section of the fabricated structure.

\subsection{Measurements}

The coupling efficiency is determined from a fiber-to-fiber transmission measurement. The structures consist of an input coupler and an output coupler, connected by a waveguide. Only the input coupler has a bottom mirror. On the sample, we have also bonded reference structures without gold mirror. In this way, we are able to evaluate the performance of the coupler with bottom mirror. A fiber, connected to a tunable laser, is positioned above the input grating at an angle of 10 degrees. Another fiber, connected to a power detector, is positioned (also at 10 degrees) above the output grating. By characterizing the losses in our setup, we are able to calculate the coupling efficiency. For the bonded couplers without mirrors, we assumed that input coupler and output coupler are the same, and the coupling efficiency is determined to be $26 \%$. We used this value for extracting the coupling efficiency of a grating coupler with bottom mirror (input coupler). The coupling efficiency $69 \%$ and the $1 \mathrm{~dB}$ bandwidth is $40 \mathrm{~nm}$. The measurement results are shown in Fig 5. 


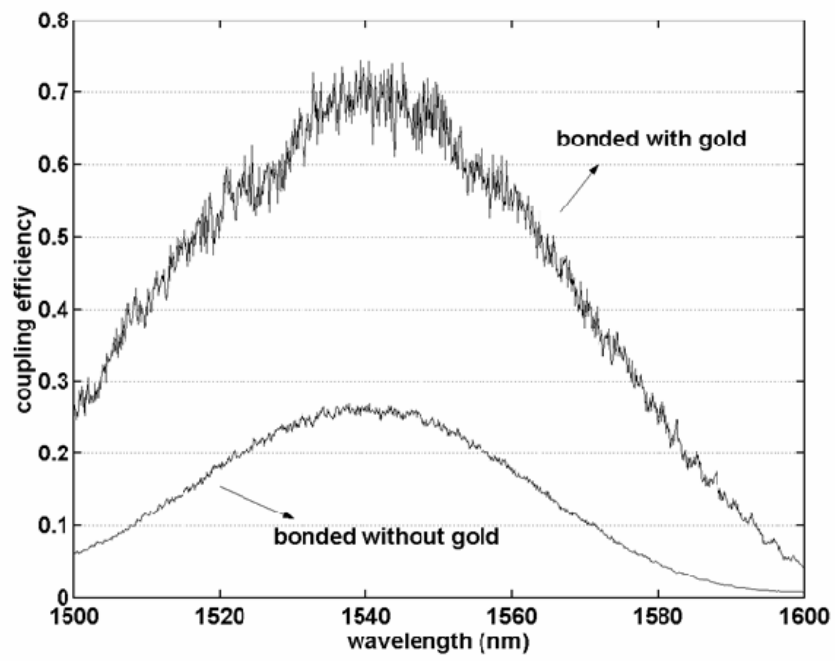

Figure.5. Measurement result for bonded SOI-grating couplers with and without bottom mirror

\section{Compact arrayed waveguide grating}

Silicon wires are very attractive to implement passive linear wavelength filters for WDM purposes, such as arrayed waveguide grating (AWG) routers, multiplexers or demultiplexers. With lower index contrast systems, the size of an AWG is limited by the bend radius. The use of Silicon wires, with a bend radius of just a few $\mu \mathrm{m}$ exhibiting negligible losses, overcomes this problem. The size of an AWG is then limited by design parameters such as the desired free spectral range and the number of wavelength channels.

\subsection{Double etch scheme: lowering the index contrast}

One of the major sources of loss in an AWG, and even more pronounced in a high index contrast material system, is scattering at the gaps between the array waveguides. We reduced the scattering loss by using a lower index contrast for the star coupler. This was done through a double etch scheme, where the waveguides around the star coupler were etched only $70 \mathrm{~nm}$ deep in the $220 \mathrm{~nm}$ thick Si layer. A double taper approach converts between the $220 \mathrm{~nm}$ deeply etched waveguides at the inputs, outputs and in the array. This is illustrated in Fig. 6

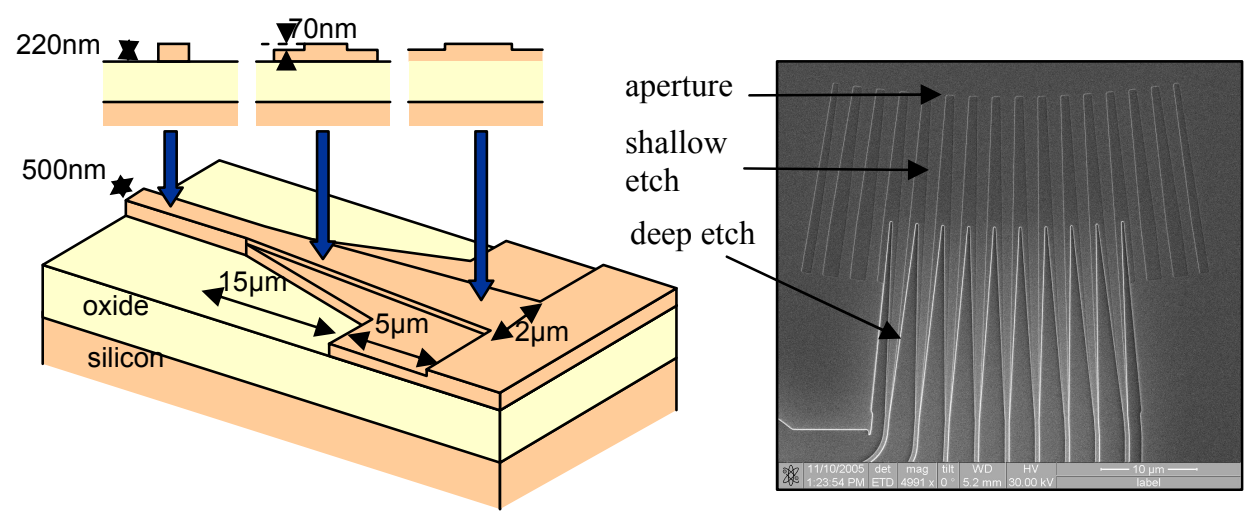

Figure 6: Double etch scheme used at the star couplers (left) schematic (right) SEM picture of input side of star coupler 


\subsection{Reducing phase errors}

The second point tackled is the crosstalk arising from multiple sources. The high-index contrast, submicron size waveguides are very sensitive to small waveguide width variations such as random roughness and longer scale variations or more systematic mask digitization errors. This gives rise to phase errors which can be very significant, up to a considerable fraction of $\pi$. We lowered this sensitivity by using broader waveguides, for which the effective index changes less with waveguide width. As these waveguides are multimode, more narrow waveguides are still used for the bends and near the tapers at the star couplers. Figure 7 illustrates the principle. For large phase errors, a crosstalk reduction of up to $10 \mathrm{~dB}$ can be achieved. Other contributions to the crosstalk are the truncation of the field due to the finite array aperture and other aberrations which can be stronger in this high index contrast, wide angle system.
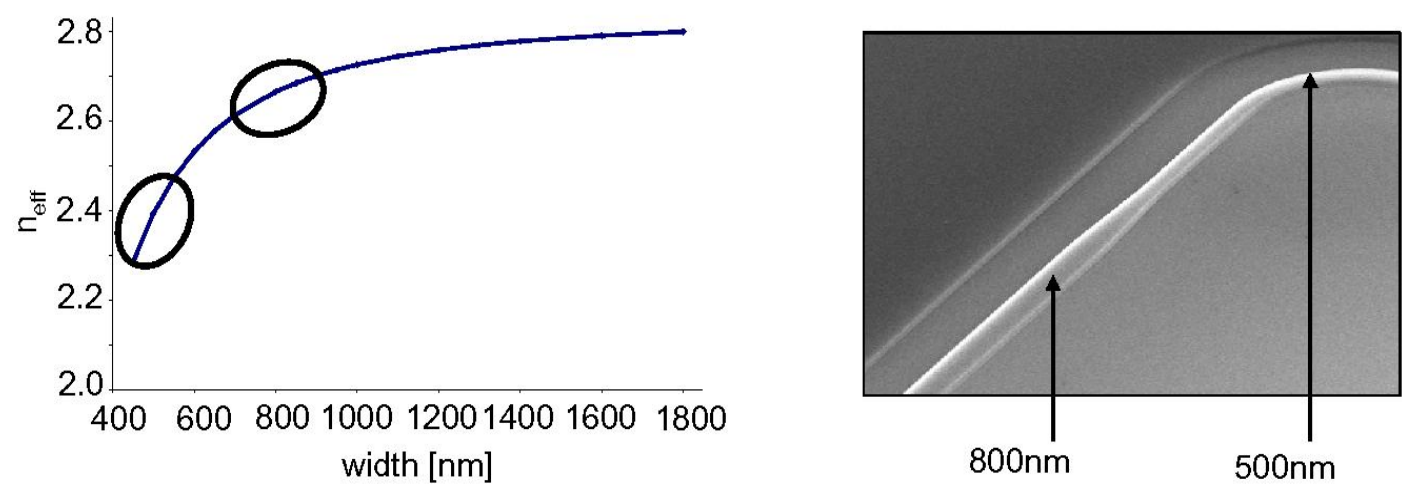

Figure 7: left: Effective index as a function of wire width. right: Using broader waveguides with less phase errors, except in bends. A linear taper converts between broad and narrow waveguides

\subsection{6 channel AWG}

Here we present a 16 channel AWG for routing purposes, with a $200 \mathrm{GHz}$ channel spacing. The AWG has 36 arrayed waveguides. The gaps between the (shallowly etched) array waveguide apertures are $190 \mathrm{~nm}$. The device has an insertion loss of 2 to $3 \mathrm{~dB}$, and a sidelobe level (giving rise to crosstalk) of -15 to $-20 \mathrm{~dB}$. The global insertion loss nonuniformity (of the outer channels compared to the inner channels) results from the router design, with a free spectral range of $3.2 \mathrm{THz}$. 

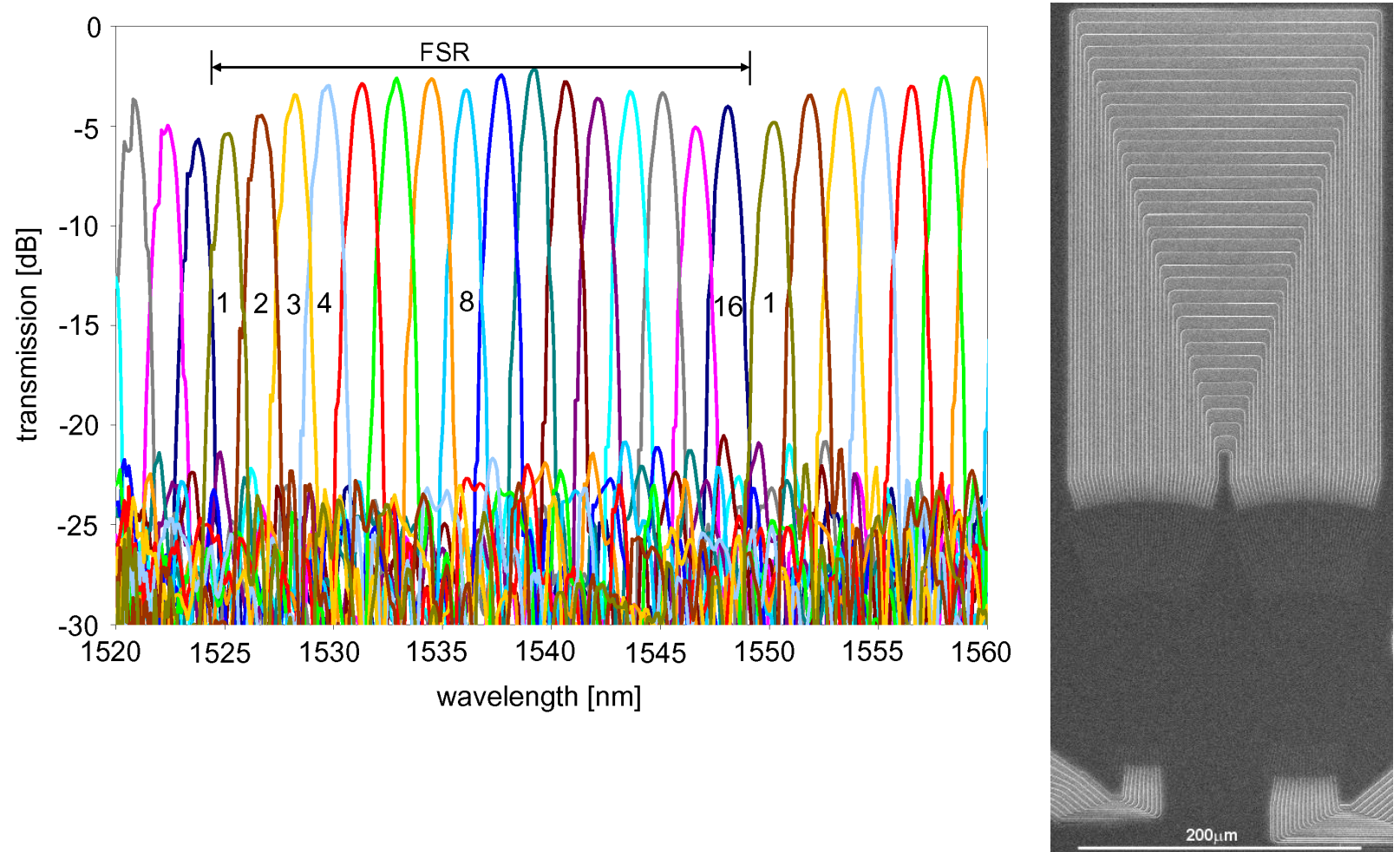

Figure 8: 16 channel (200GHz) AWG (left) transmission spectra from central input to all outputs (right) SEM picture

\subsection{A fiber pigtailed AWG}

An AWG with 4 inputs and 4 outputs was coupled to fiber using a commercially available eight fiber array connector and vertical fiber coupling. The AWG was designed as a router with a $250 \mathrm{GHz}$ channel spacing and $1 \mathrm{THz}$ free spectral range, with a $425 \times 155 \mu \mathrm{m}^{2}\left(<0.07 \mathrm{~mm}^{2}\right)$ footprint. Ten arrayed waveguides were used, and the star couplers used the double etch scheme. The arrayed waveguides were not broadened to $800 \mathrm{~nm}$ as in the AWG in Fig. 8.

The connector consists of a $\mathrm{V}$-groove assembly with standard single mode fibers spaced $125 \mu \mathrm{m}$ apart. The connector is first aligned passively to a spare set of grating couplers using alignment markers on chip. These couplers are interconnected two by two by alignment waveguides. Due to the large alignment tolerances, transmitted power can be detected after passive alignment already. Further optimization is done by active alignment on these interconnected couplers. The mask layout and concept are shown in Fig. 9.

The fibers are mounted under a $9^{\circ}$ angle in order to avoid reflections at the waveguide facet and optimize transmission. The connector facet is polished under an $8^{\circ}$ angle, so that a $1^{\circ}$ angle remains between connector facet and chip. In this way the connector edge can rest on the chip without harming the structures. The connector is fixed to the chip by UV curable glue with a low shrinkage.

As the connector surface is about the same size as the die size, the result is a mechanically attractive approach. The component is just a connector with a chip attached to it. The die size without passive alignment structures is about $2 \mathrm{~mm}^{2}$ 


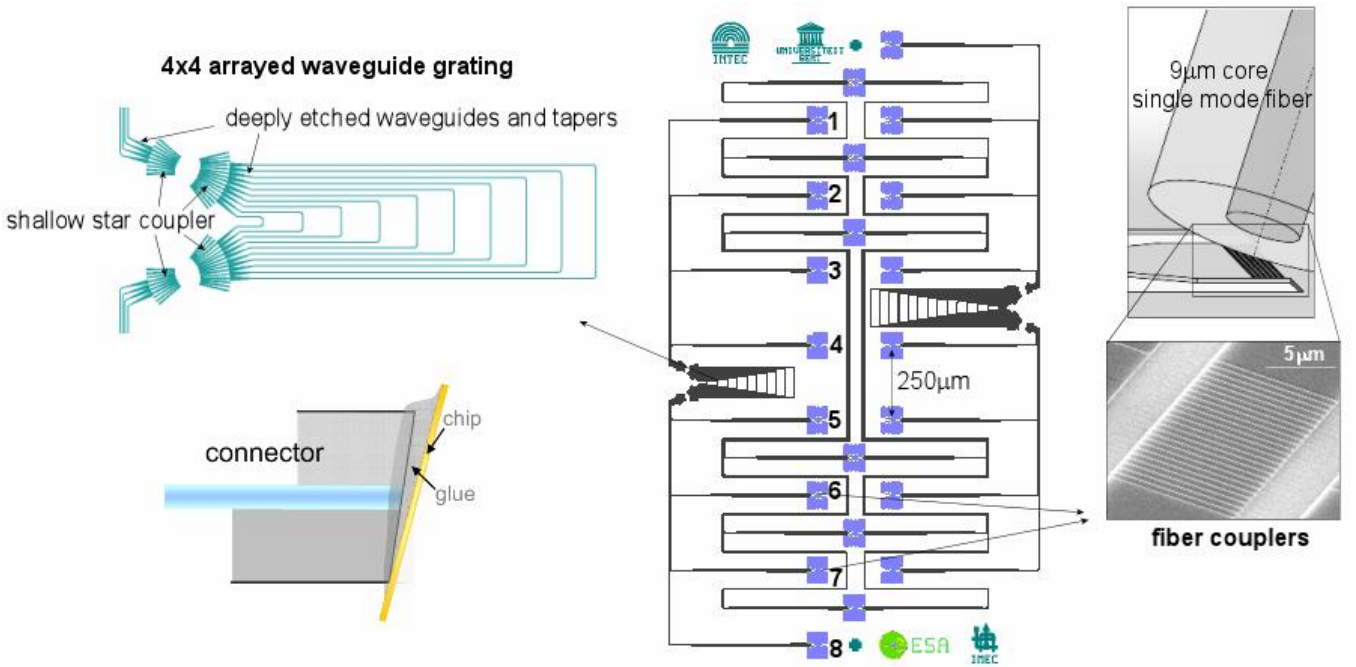

Figure 9: A fiber pigtailed $A W G$, with pigtailing concept, $A W G$, mask layout with fiber couplers (1-8) and 6 spare fiber couplers interconnected two by two.

Figure 10 shows the measured transmission spectra of the fiber coupled component. The pigtailed component has a fiber to fiber insertion loss of $12.5 \mathrm{~dB}$ and a sidelobe extinction of $-12 \mathrm{~dB}$. The insertion loss of the AWG as such was measured to be about $3.5 \mathrm{~dB}$, which mainly consists of losses in the star couplers. Over a 2 months period after gluing, the spectra experienced an almost negligible change. The fiber coupling efficiency can be enhanced further through an optimized design. However, here we focused on easy fabrication with CMOS based processes and limited number of fabrication steps. The sidelobe level can be reduced partly through a better design and using broader waveguides.
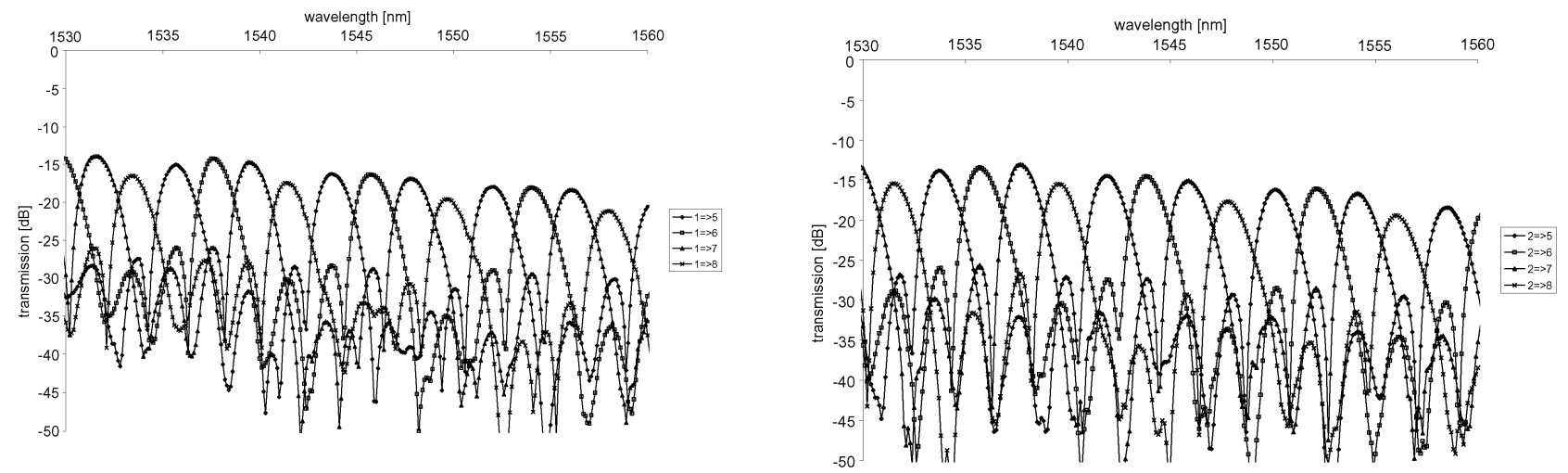

Figure 10: Overlaid fiber to fiber transmission spectra from input ports 1 (left) and 2 (right) to all 4 output ports.

The component only works for TE polarization as both the waveguides and grating couplers are highly polarization dependently. However, the connector is also available with PM fiber. Additionally, in theory one can use polarization diversity in order to achieve polarization independent operation.

\subsection{Application in a 10Gbit/s reconfigurable backplane interconnect}

While the loss and sidelobe extinction figures are still large compared to commercially available components, they already allow for deployment in some systems with limited scalability. The fiber pigtailed component described in was used as the core of a high-speed, reconfigurable backplane interconnect, which interconnects 4 nodes. This system was a demonstrator prototype for high speed optical backplanes in telecom and observation satellites. The pigtailed AWG was temperature controlled around room temperature and the inputs were polarization controlled. Each node consisted of an FPGA based board implementing a basic level of hybrid distributed/centralized network control and the generation, transmitting, receiving and bit by bit comparison of $10 \mathrm{Gbit} / \mathrm{s}$ randomly generated data. The boards 
employed laserless transponders with a $10 \mathrm{Gbit} / \mathrm{s}$ modulator and a high sensitivity receiver, and two of the boards were equipped with fast tunable lasers with $50 \mathrm{GHz}$ spaced wavelength channels between $1528 \mathrm{~nm}$ en $1563 \mathrm{~nm}$. By doing a bit by bit comparison of sent and received data, a direct count of the bit errors and thus the bit error rate (BER) was obtained. Figure 11 illustrates the demonstrator and measurement setup. The power penalty due to single channel crosstalk in the router was measured by comparing a back to back measurement of the bit error rate as a function of received power, and a second measurement with a second crosstalk generating communication channel. Depending on the input/output combination, crosstalk varied greatly with the SOI component, depending on the exact alignment of the signal wavelengths with the sidelobes. For a BER of $10^{-10}$, incoherent crosstalk resulted in a power penalty of 0.1 to $1 \mathrm{~dB}$ for crosstalk levels ranging from -19 to $-11.1 \mathrm{~dB}$. Coherent crosstalk resulted in power penalties of 0.2 to $2 \mathrm{~dB}$ for crosstalk levels between -20.1 to $-14.1 \mathrm{~dB}$. Characterization of the demonstrator supported a model in which the penalty grows linearly when more additive crosstalk generating channels are added. However, beyond a power penalty of $5 \mathrm{~dB}$, we consider this model not to be valid anymore. As a result, within the power budget of a realistic system of $20 \mathrm{~dB}$ ( $2 \mathrm{dBm}$ modulated output power, $-18 \mathrm{dBm}$ sensitivity), the figures indicate a scalability of the SOI component of just 4 to 9 nodes at a BER of $10^{-10}$. Therefore, even at the current performance level, the SOI components can have a realistic application, albeit with a limited port count. The scalability can be enhanced by lowering the fiber coupling losses and the crosstalk. An AWG such as the one in Fig. 8 would already have a scalability of around 20 ports.
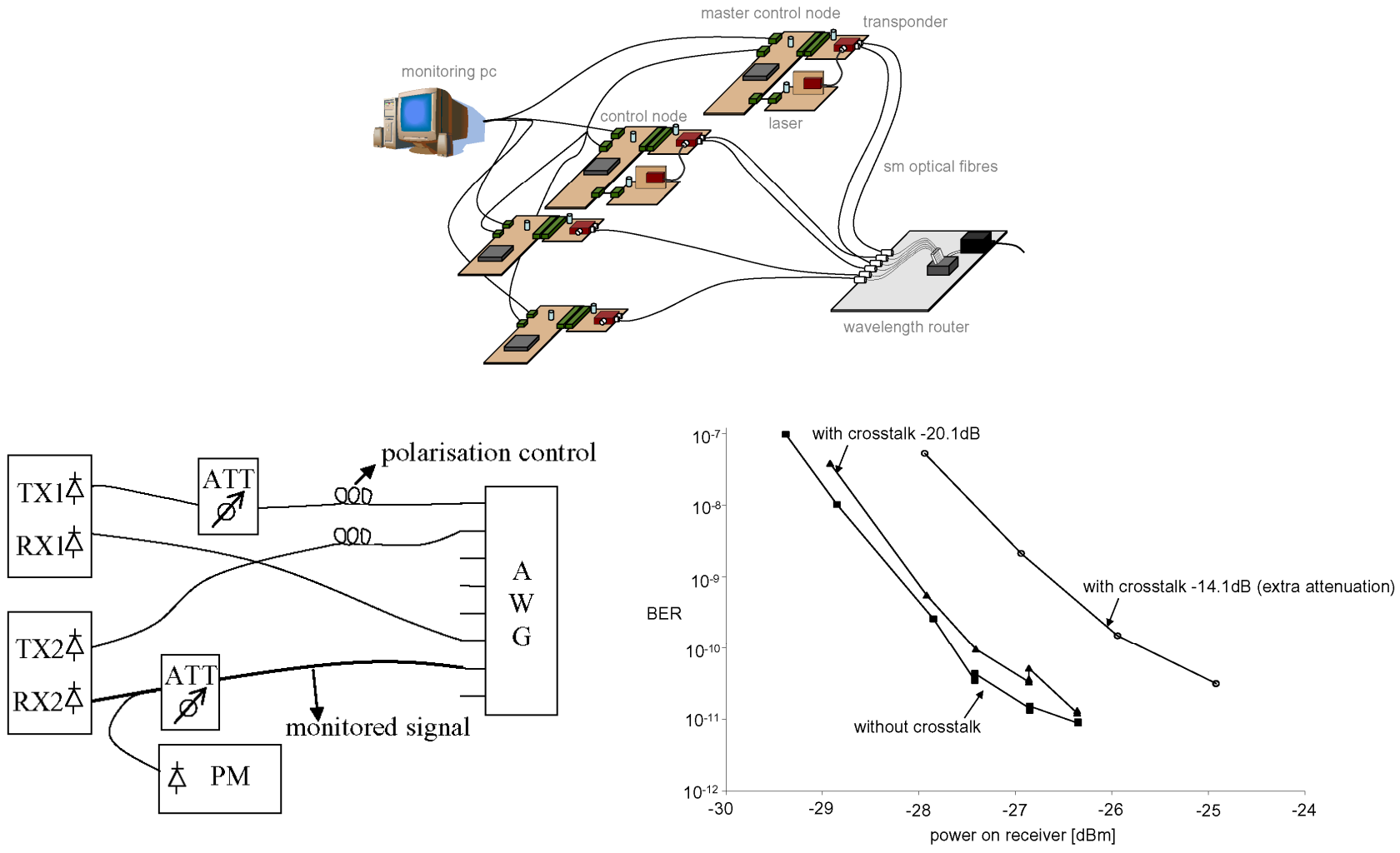

Figure 11: Four node demonstrator with SOI component, fast tunable lasers, laserless transponders and electrical control and data processing nodes. Top: schematic. Bottom left: power penalty measurement setup. Bottom right: example of a power penalty measurement, for coherent crosstalk. The attenuator in the input port is used to further attenuate the signal. The attenuator in the output port is used to vary the power incident on the receiver and thus the bit error rate. 


\section{RING RESONATOR BASED BIOSENSOR}

Sensing of biomolecules is gaining interest due to its applications in many areas such as bacterial and virus detection, medical diagnostics, drug development, food and environmental control. Most commercialized biosensors rely on detection of labeled molecules. However, there is a growing need for label-free detection methods for fast, sensitive and quantitative sensing. We propose a highly miniaturized label-free biosensor based on optical microcavities in Siliconon-Insulator (SOI). SOI offers a high refractive index contrast suitable for the fabrication of nanophotonic circuits including micron- and submicron sized optical cavities of very high quality. The shift of resonance wavelength that occurs when the dielectric surroundings of such a cavity is changed, can be used for sensing.
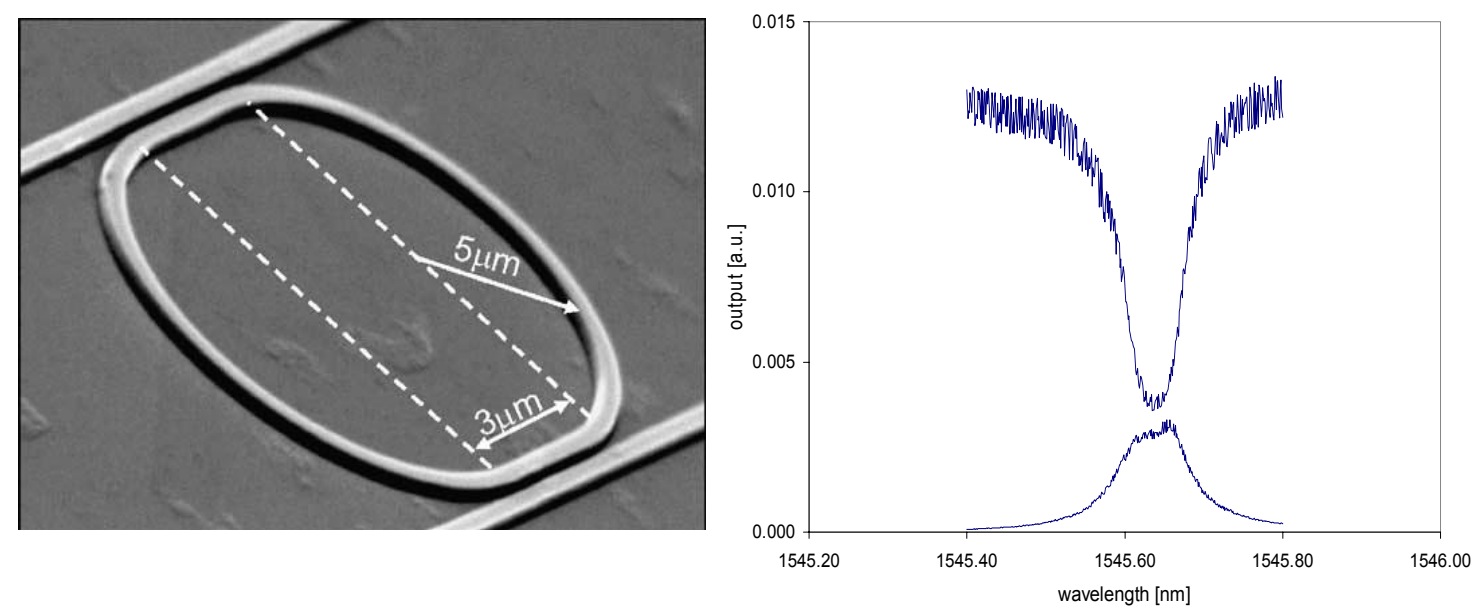

Figure 12 SEM picture and pass-drop spectra of a 5 micron microring resonator.

We demonstrate a sensor based on an SOI optical microring resonator with radius 5 micron. Q factors over 20,000 are easily achievable with our fabrication process and optimized design, see Figure 12 for pass and drop spectra of the resonator. We find our device capable of detecting bulk refractive index changes of $7 * 10^{-5}$ using salt concentrations (see figure 13). 

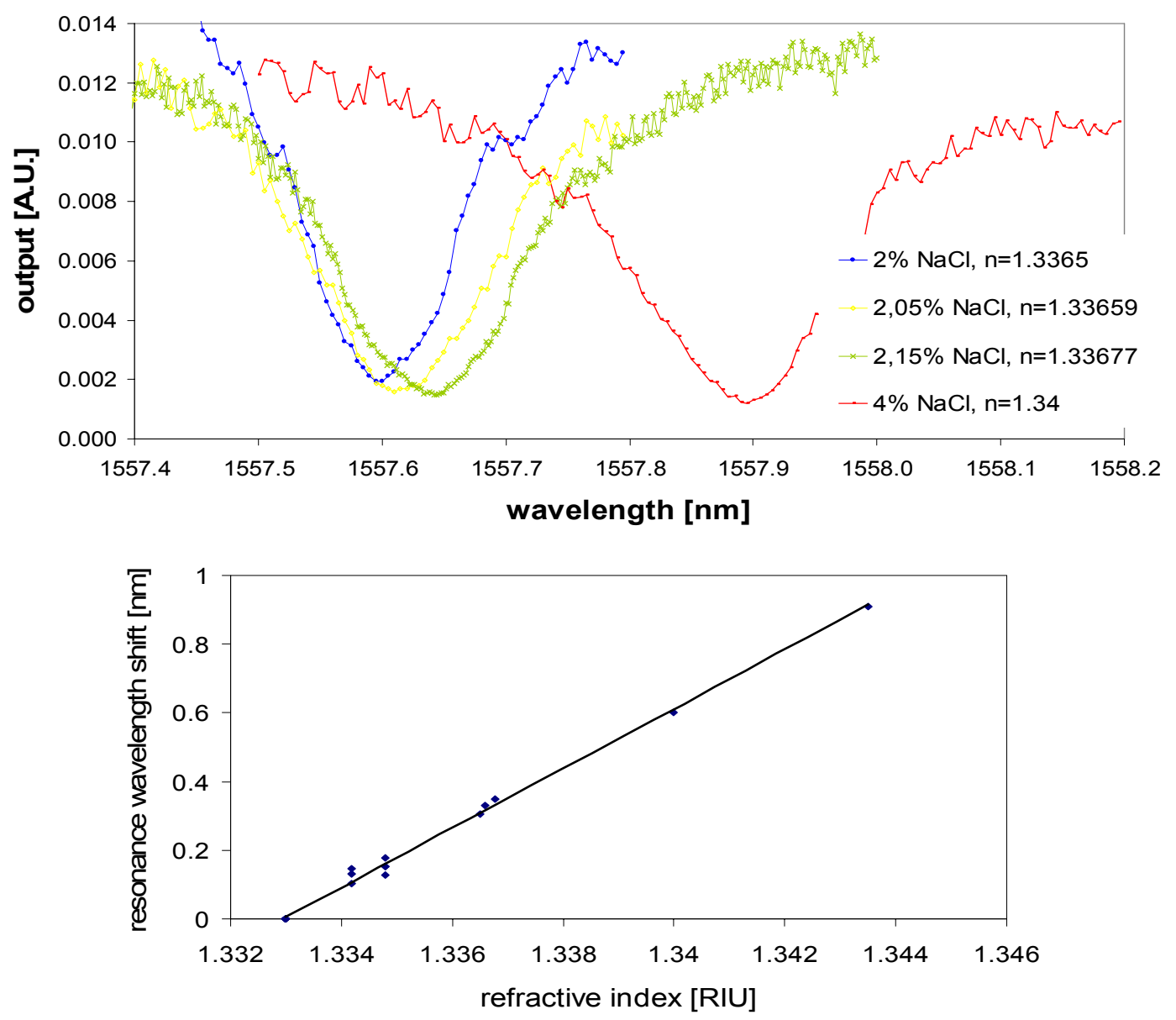

Figure 13 Measured resonance wavelength shift using different salt concentrations for bulk refractive index sensing.

Modification of the semiconductor surface, allowing for immobilization of biomolecules, is characterized by X-ray photoelectron spectroscopy, ellipsometry and Atomic Force Microscopy (AFM). We test avidin/biotin affinity sensing by flowing an avidin solution over the modified microring and observe the corresponding resonance wavelength shift, as illustrated in Fig. 14. Moreover, the use of grating couplers as explained elsewhere in this article allows for easy and alignment tolerant coupling of light into and out of our device. Different negative control configurations, using aspecific binding molecules or non matching biorecognition elements, prove the realization of real specific binding of the avidinbiotin couple. The sensor is fabricated by means of standard technologies, in particular deep-UV lithography, used for the fabrication of very large scale integrated (VLSI) electronic circuits. These technologies allow for high levels of integration indicating the potential of multiparameter analysis and lab-on-chip applications. Moreover, they allow for high throughput fabrication as needed for high volume applications. Further miniaturization will decrease the sensing area, allowing for detection of extremely low analyte concentrations while lining up the microrings in arrays will allow for cheap high throughput label-free multiparameter analyses. 


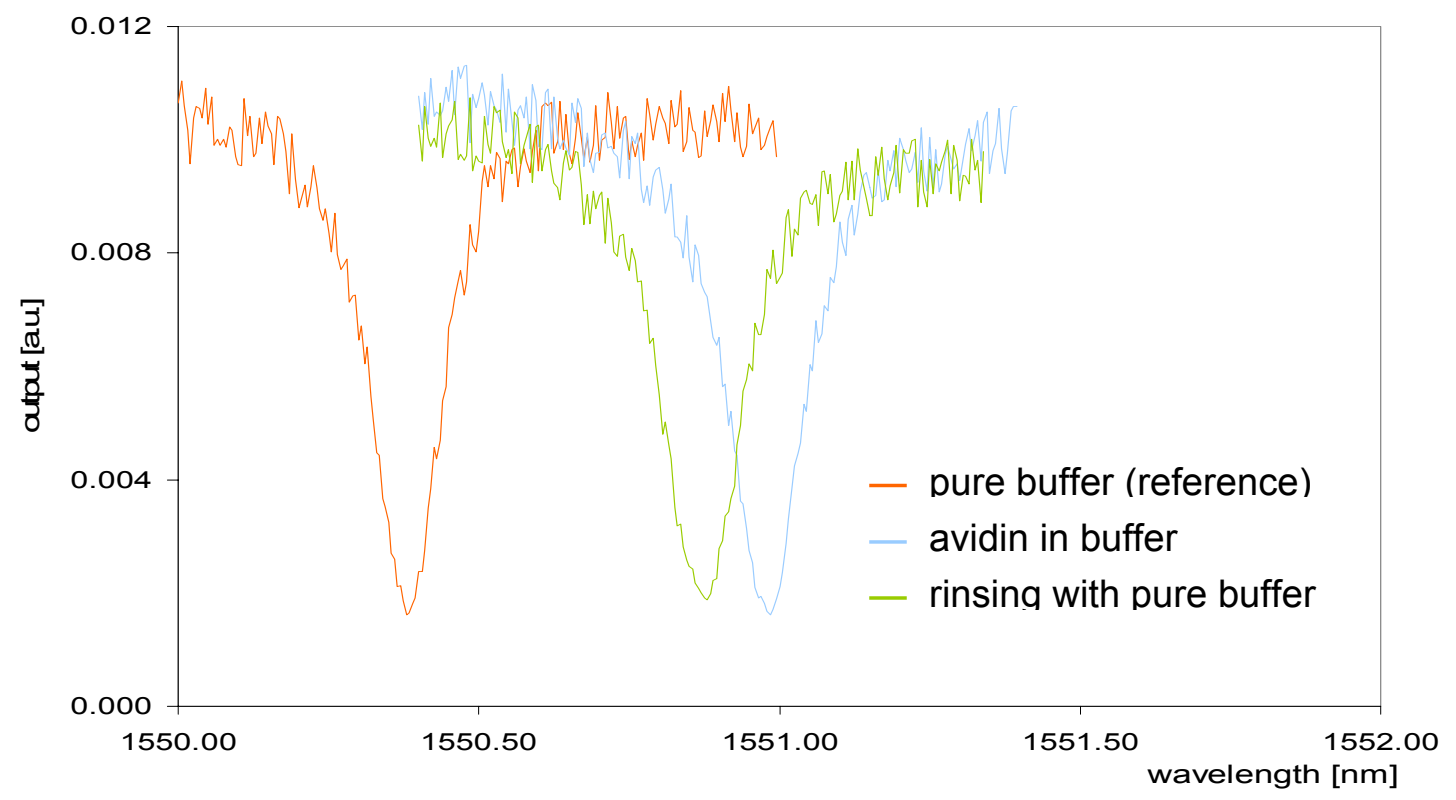

Figure 14 Illustration of biotin/avidin sensing : Binding of $0.5 \mathrm{mg} / \mathrm{ml}$ avidin concentration to a biotinylated surface.

\section{CONCLUSIONS}

We presented recent progress in several devices based on silicon-on-insulator nanophotonics using deep-UV lithography. We reported on high efficiency grating couplers, ultra-compact arrayed waveguide gratings and ringresonator based biosensors.

\section{ACKNOWLEDGEMENTS}

PD, FVL and KDV thank the Institute for the Promotion of Innovation through Science and Technology in Flanders (IWT-Vlaanderen) for a specialization grant. PB acknowledges support from the Flemish Fund for Scientific Research (FWO Vlaanderen). Parts of this work were performed in the context of the Ghent University GOA biosensor project, the Belgian projects IAP Photon and IWT epSOC, the European network of excellence ePIXnet. and the ESA/ESTEC contract 17844/03/NL/HE. We also thank J. Wouters, S. Beckx and P. Jaenen for SOI device processing, I. Bartolozzi and E. Schacht for functionalising the biosensors. T. Farell and N. Ryan from Intune Technologies, E.D. KyriakisBitzaros and E. Grivas from NCSR Demokritos, Greece and I. McKenzie from ESTEC/ESA for the fruitful collaboration within the the ESA multigigabit optical backplane interconnections project.

\section{REFERENCES}

1. T. Tsuchizawa, K. Yamada, H. Fukuda, T. Watanabe, J. Takahashi, M. Takahashi, T. Shoji, E. Tamechika, S. Itabashi, H. Morita, "Microphotonics Devices Based on Silicon Microfabrication Technology", IEEE J. Select. Top. Quant. Elect 11, 232-240 (2005)

2. G. Roelkens, P. Dumon, W. Bogaerts, D. Van Thourhout, R. Baets, "Efficient Silicon-on-Insulator fiber coupler fabricated using $248 \mathrm{~nm}$ deep UV lithography", IEEE PTL 17, 2613-2615 (2005). 
3. D. Tailaert, R. Baets, P. Dumon, W. Bogaerts, D. Van Thourhout, B. Luyssaert, V. Wiaux, S. Beckx, J.Wouters, "Silicon-on-Insulator Platform for Integrated Wavelength-Selective Components," in Proc. of 2005 IEEE/LEOS Workshop on Fibres and Optical Passive, Italy, 2005, pp. 115-120.

4. I. Christiaens, G. Roelkens, K. De Mesel, D. Van Thourhout, R. Baets, "Thin film devices fabricated with BCB waferbonding," IEEE JLT 23, 517-523 (2005).

5. P. Bienstman and R. Baets, "Optical modelling of photonic crystals and VCSELs using eigenmode expansion and perfectly matched layers," Optical and Quantum Electronics 33, 327-341 (2001).

6. W. Bogaerts, R. Baets, P. Dumon, V. Wiaux, S. Beckx, D. Taillaert, B. Luyssaert, J. Van Campenhout, P. Bienstman, and D. Van Thourhout, "Nanophotonic waveguides in silicon-on-insulator fabricated with CMOS technology," IEEE PTL 23, 401-412 (2005). 


\section{Asia-Pacific Optical Communications 2006}

3-7 September 2006

Gwangju, South Korea

\section{APO $200 \%$

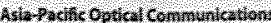

\section{PROCEEDINGS OF SPIE ON CD-ROM}

\section{Asia-Pacific Optical Communications 2006}

\section{3-7 September 2006 Gwangiu, South Korea}

Volumes $6351,6352,6353,6354,6355$

6351: Passive Components and Fiber-based Devices III

6352: Optoelectronic Materials and Devices

6353: Oplical Transmission, Switching, and Subsystems IV

6354: Network Architecłures, Management, and Applications IV

6355: Advanced LEDs for Solid State Lighting 